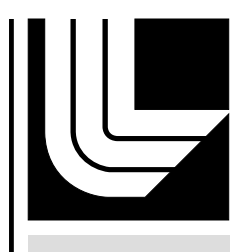

LAW REN CE LIVERMORE N A TION A L LABORATORY

\title{
UCRL-TR-209928
}

\section{Orbiter Composite Overwrapped Pressure Vessels}

Steve DeTeresa

February 24, 2005 
This document was prepared as an account of work sponsored by an agency of the United States Government. Neither the United States Government nor the University of California nor any of their employees, makes any warranty, express or implied, or assumes any legal liability or responsibility for the accuracy, completeness, or usefulness of any information, apparatus, product, or process disclosed, or represents that its use would not infringe privately owned rights. Reference herein to any specific commercial product, process, or service by trade name, trademark, manufacturer, or otherwise, does not necessarily constitute or imply its endorsement, recommendation, or favoring by the United States Government or the University of California. The views and opinions of authors expressed herein do not necessarily state or reflect those of the United States Government or the University of California, and shall not be used for advertising or product endorsement purposes.

This work was performed under the auspices of the U.S. Department of Energy by University of California, Lawrence Livermore National Laboratory under Contract W-7405-Eng-48. 


\section{Orbiter Composite Overwrapped Pressure Vessels}

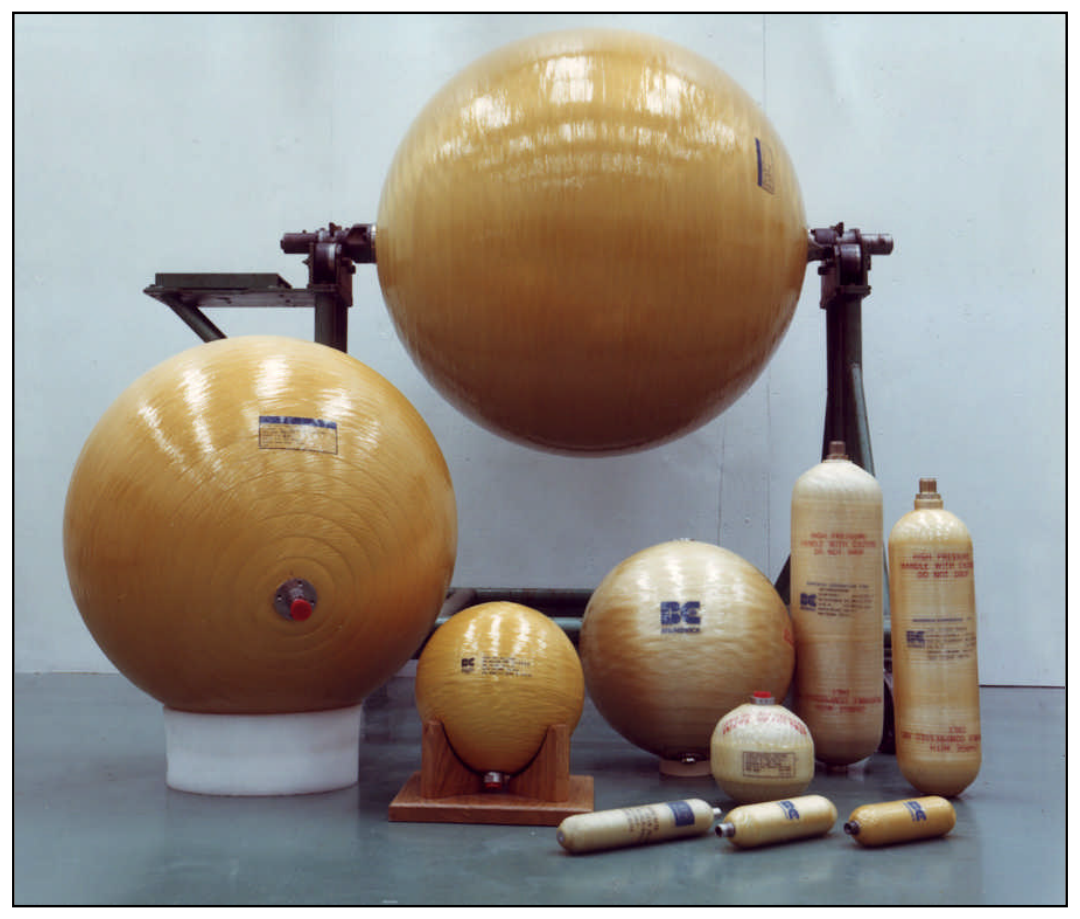

Steve DeTeresa

February 22, 2005 


\section{Pressure Histories: Joint DOE/DuPont Study}

\begin{tabular}{|c|c|c|c|c|c|c|c|c|}
\hline \multirow[t]{6}{*}{ Comment } & $\begin{array}{l}\text { Spool B } \\
814133\end{array}$ & Vessel \# & $\begin{array}{l}\text { Failure Time } \\
\mathrm{hr}\end{array}$ & Comment & $\begin{array}{l}\text { Spool C } \\
815123\end{array}$ & Vessel \# & $\begin{array}{l}\text { Failure Time } \\
\mathrm{hr}\end{array}$ & Comment \\
\hline & & & & & Rank & & & \\
\hline & 1 & 11 & 1,495 & & 1 & 13 & 964 & \\
\hline & 3 & 33 & 3,122 & & 3 & 111 & 1,400 & 1,400 to 2,137 \\
\hline & 4 & 17 & 3,150 & 3,150 to 3,653 & 4 & 45 & 1,527 & \\
\hline & 5 & 59 & 3,685 & & 5 & 115 & 2,907 & \\
\hline \multirow{10}{*}{$\begin{array}{l}1,416 \text { to } 1,563 \\
1,450 \text { to } 1,822\end{array}$} & 6 & 118 & 4,299 & & 6 & 57 & 3,122 & 3,122 to 3,624 \\
\hline & 9 & 86 & 9,399 & & & & & \\
\hline & & Mean Spool B & 4,557 & & & Mean Spool C & 2,215 & \\
\hline & & Std Dev & 2,615 & & & Std Dev & 1,051 & \\
\hline & & CV (\%) & $57.4 \%$ & & & CV (\%) & $47.4 \%$ & \\
\hline & & Median & 3,685 & & & Median & 2,217 & \\
\hline & & Range Spool B & 7,904 & & & Range Spool C & 2,517 & \\
\hline & & Range/Mean & 1.73 & & & Range/Mean & 1.14 & \\
\hline & & Range/Median & 2.14 & & & Range/Median & 1.14 & \\
\hline & & MinTime/Mean & 0.33 & & & MinTime/Mean & 0.44 & \\
\hline
\end{tabular}




\section{Pressure Histories: Joint DOE/DuPont Study}

\begin{tabular}{|c|c|c|c|c|c|c|}
\hline Comment & $\begin{array}{l}\text { Spool E } \\
889234\end{array}$ & Vessel \# & $\begin{array}{c}\text { Failure Time } \\
\mathrm{hr}\end{array}$ & Comment & All Spools & \\
\hline \multirow{18}{*}{3,644 to 4,056} & Rank & 117 & 418 & & & \\
\hline & 2 & 119 & 1,404 & & & \\
\hline & 3 & 32 & 1,790 & & & \\
\hline & 4 & 34 & 2,304 & 2,304 to 2,448 & & \\
\hline & 5 & 120 & 3,213 & & & \\
\hline & 6 & 114 & 3,412 & & & \\
\hline & 7 & 5 & 3,600 & & & \\
\hline & 8 & 116 & 4,195 & & & \\
\hline & 9 & 43 & 4,336 & & & \\
\hline & 10 & 110 & 6,650 & & & \\
\hline & & Mean Spool E & 3,132 & & Mean (All) & 2,856 \\
\hline & & Std Dev & 1,767 & & Std Dev & 2,056 \\
\hline & & CV (\%) & $56.4 \%$ & & CV (\%) & $72.0 \%$ \\
\hline & & Median & 3,313 & & Median & 2,304 \\
\hline & & Range Spool E & 6,232 & & Range (All) & 8,981 \\
\hline & & Range/Mean & 1.99 & & Range/Mean & 3.14 \\
\hline & & Range/Median & 1.88 & & Range/Median & 3.90 \\
\hline & & MinTime/Mean & 0.13 & & MinTime/Mean & 0.15 \\
\hline
\end{tabular}




\section{Pressure Histories: 3,380 psi vessels}

$\begin{array}{cccrrc}\text { Rank } & \text { Vessel \# } & \text { Spool } & \text { Time to Failure, hr } & \text { Time to Failure, yr } & \begin{array}{c}\text { Comment } \\ 1\end{array} \\ 204 & 7 & 3,960 & 0.45 & \text { Used Lower Failure Time } \\ 2 & 218 & 7 & 5,376 & 0.61 & \\ 3 & 163 & 6 & 7,320 & 0.84 & \\ 4 & 95 & 3 & 8,615 & 0.98 & \\ 5 & 142 & 5 & 9,120 & 1.04 & \\ 6 & 68 & 2 & 14,440 & 1.65 & \\ 7 & 180 & 6 & 16,104 & 1.84 & \\ 8 & 155 & 5 & 20,231 & 2.31 & \\ 9 & 171 & 6 & 20,233 & 2.31 & \\ 10 & 134 & 5 & 35,880 & 4.10 & \\ 11 & 228 & 8 & 48,048 & 5.48 & \text { All Vessels filled } 6-27-77 \\ 12 & 250 & 8 & 52,848 & 6.03 & \\ 13 & 3 & 1 & 57,816 & 6.60 & 57,816<\text { Failure }<64,824 \\ 14 & 116 & 1 & 57,816 & 6.60 & 57,816<\text { Failure }<64,824 \\ 15 & 106 & 4 & 57,816 & 6.60 & 57,816<\text { Failure }<64,824 \\ 16 & 27 & 1 & 75,624 & 8.63 & 75,624<\text { Failure }<75,984 \\ 17 & 111 & 4 & 87,760 & 10.02 & 87,760<\text { Failure }<89,448 \\ 18 & 127 & 4 & 87,760 & 10.02 & 87,760<\text { Failure }<89,448 \\ 19 & 224 & 8 & 90,696 & 10.35 & 90,696<\text { Failure }<92,904 \\ 20 & 18 & 1 & 94,344 & 10.77 & \text { Not Failed as of } 4 / 88 \\ 21 & 117 & 4 & 94,344 & 10.77 & \text { Not Failed as of } 4 / 88\end{array}$

Date(s) of Failure

Check Failure, hr

$12 / 21 / 86$
$7 / 9 / 87$
$1-31-84$ to $11-19-84$
$1-31-84$ to $11-19-84$
$1-31-84$ to $11-19-84$
$2-11-86$ to $2-26-86$
$5-21-87$ to $9-10-87$
$5-21-87$ to $9-10-87$
$11-87$ to $2-88$

\section{$12 / 21 / 86$}

$1-31-84$ to $11-19-84$

$1-31-84$ to $11-19-84$

- 11 to $11-19-84$

$5-21-87$ to $9-10-87$

9-10-87

Not Failed as of $4 / 88$
48,048

52,848

57,816

57,816

57,816

75,624

86,760

86,760

90,696

94,344 


\section{Pressure Histories: 3,380 psi vessels}

Statistics
Mean (All)
Std Dev
CV (\%)
Median
Range (All)
Range/Mean
Min time/Mean

Mean w/o \#7

Std Dev

CV (\%)

Median

Range w/o \#7

Range/Mean

Min time/Mean

Mean 2,3,5,6

Std Dev

CV (\%)

Median

Range 2,3,5,8

Range/Mean

Min time/Mean

Mean 1,4,8

Std Dev

CV $(\%)$

Median

Range 1,4,8

Range/Mean

Min time/Mean

\section{hours}

45,055

33,526

74.41

48,048

90,384

2.01

0.09

49,306

32,381

65.67

52,848

87,024

1.76

0.15

16,493

9,325

56.54

15,272

28,560

1.73

0.44

73,170

18,404

25.15

75,624

46,296

0.63

0.66 years

5.14

3.83

74.41

5.48

10.32

2.01

5.63

3.70

65.67

6.03

9.93

1.76

1.88

1.06

56.54

1.74

3.26

1.73

8.35

2.10

25.15

8.63

5.28

0.63
Used Lower Failure Time

In Each Case

21 Total Vessels

19 Failed

Used Lower Failure Time

In Each Case

8 Total Vessels

All Failed

\author{
11 Total Vessels \\ 9 Failed \\ 2 Intact After 10.77 years
}

